\title{
Change in the physical parameters of rocks due to hydrofracturing
}

\author{
Henryk Marcak ${ }^{1, *}$ \\ ${ }^{1}$ Polish Academy of Sciences, Institute of Geophysics, 01-452 Warszawa, Poland
}

\begin{abstract}
Hydrofracturing is a procedure performed for the activation and intensification of fluid flow in shale and geothermal deposits. This operation involves the risk of inducing strong seismic shocks and the leakage of borehole liquids into drinking water reservoirs. The changes of the physical properties of the rocks deformed by hydro-fracturing are modelled numerically. The structure of the model is presented in the paper. It is also demonstrated that the development of fracture zones resulting from the hydro-fracturing process can be investigated by electromagnetic and seismic methods. The flow of liquids through linear elements (fractures and cracks) increases the risk of destructive action through a combination of tectonic and hydro-fracture stresses.
\end{abstract}

\section{Introduction}

In the hydrofracturing process, high pressure liquid is introduced into the horizontal part of a borehole forcing the liquid against the rock and causing rock fracturing, cracking the rock surrounding it, until it finally facilitates the flow of reservoir fluids, in particular gas, into the borehole. According to IMS Global Insight and Drilling Info Inc., nearly half of the holes currently drilled in the US for oil and $2 / 3$ for gas are hydro-fractured.

This method of hydrocarbon exploitation needs to be controlled. There are cases, rather rare, in which hydrofracturing produces strong seismic events. Examples include such seismic events as those which occurred during the hydro-fracturing of geothermal deposits in Basel [8]. Despite the fact that the injection of water into the rock had ceased and the water was even being pumped out, a number of seismic shocks, with increasing energy, were induced and threatened the city. During the hydrofracturing of wells in the Horn River Basin, Canada, a tremor of magnitude 3.8 was generated. There is also a threat to the public due to leakage of reservoir fluids into drinking water reservoirs as a result of hydrofracturing.

In this situation, it would be useful to have geophysical methods which allow one to control and predict the development of danger situations during hydrofracturing processes. However, geophysical methods allow one to recognise changes in the physical properties of rocks and a feasibility study on geophysical methods should be preceded by an analysis of the physical changes in the rocks resulting from hydrofracturing.

\footnotetext{
*Corresponding author: marcak@agh.edu.pl
} 


\section{Geomechanical aspects of the hydrofracturing process}

Hydrofracturing is a result of introducing pressure exceeding the formation pore pressure into a horizontal part of a borehole for a sufficiently long period of time (Fig. 1).This effect is achieved by the installation of inflatable packers in the wellbore sections which close and seal them. Water under high pressure with sand and chemicals which is pumped through the packer for a long period of time produces a vertical fracture.

Two phases of the geo-mechanical deformations effecting changes in the physical properties of the medium are important. One of these is the formation of vertical fractures in the rock. The second is the spread of cracked zones around the vertical cracks. Both of these are discussed separately.

The Griffith model can be used in a simplified description of the first phase. Due to the symmetry introduced by a horizontal borehole, a two-dimensional description can be sufficient to describe the stresses in rocks under hydro-fracturing. The stress tensor in this system has three components $\sigma_{\mathrm{x}}, \sigma_{\mathrm{y}}$ and $\tau_{\mathrm{x}, \mathrm{y}}$. By rotation of the coordinate system two main stresses can be estimated $\sigma_{\mathrm{I}}$ and $\sigma_{\mathrm{II}}$.

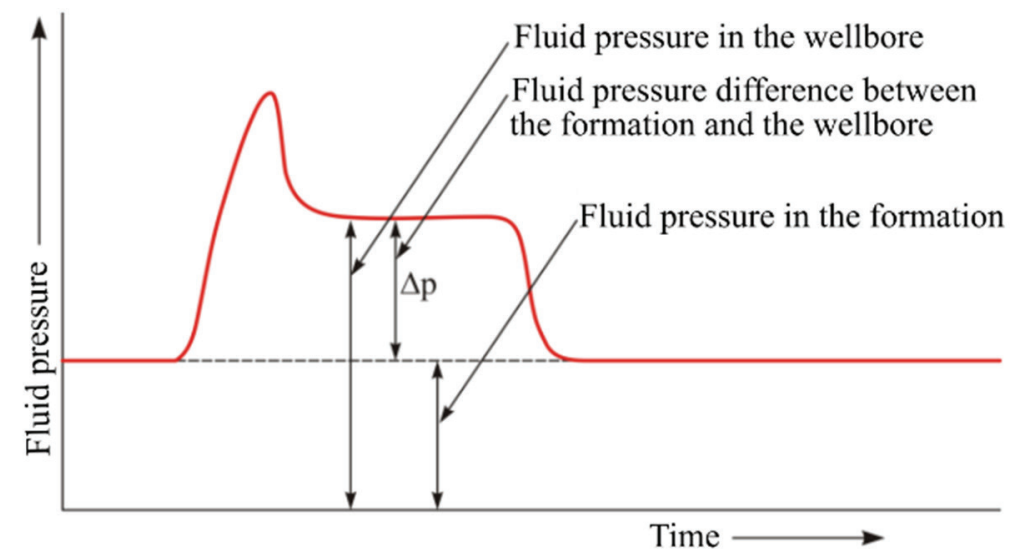

Fig. 1. Changes of pressure in rocks under hydrofracturing.

Mohr's circles are constructed in a coordinate system, ith axes for compressive stress $\sigma$ and shear stress $\tau$. Circles are drawn with centres on the $\sigma$ axes at a point $\left(\sigma_{I}+\sigma_{\text {II }}\right) / 2$ and with radius $\left(\sigma_{\mathrm{I}}-\sigma_{\mathrm{II}}\right) / 2$.

a)

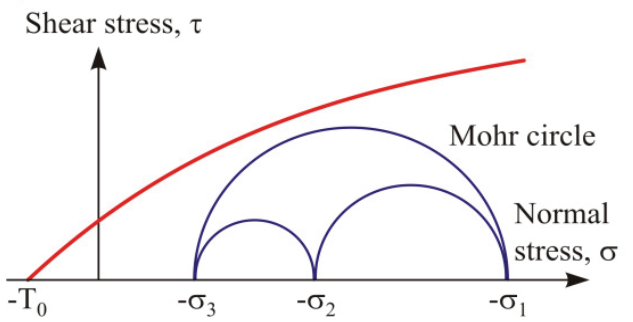

b)

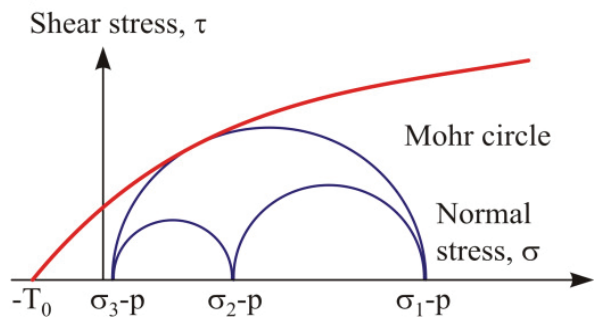

Fig. 2. Effect of hydraulic pressure on the change of stress in Mohr's circles and the Coulomb failure envelope.

The circle is shown in Figure 2a. The failure envelope (red line in Fig. 2) allows one to determine the shear stress which represents the limits of rock strength. If the medium is 
subjected to hydraulic pressure, the principal stresses in the graph are reduced by this value p. As shown in Figure $2 b$, this results in crack formation for the same values of principal stress that were too weak to produce cracking without hydraulic pressure.

Figure 3 shows the distributions of the radial, tangential and shear stresses around a circular opening in a two-axial stress system. The following values are assumed: vertical stress $\sigma_{\mathrm{z}}$ equal to $1000 \mathrm{MPa}$, Poisson's ratio $v$ equal to 0.25 . In Fig. 3, an intensive increase of the tensile stress can be noted in the roof and bottom of the opening. This example explains why the crack extends in a direction perpendicular to the minimum main stresses.

There are many papers outlining the geo-mechanical conditions for extending fractures as a result of hydrofracturing. The classical one is the so-called two-dimensional model of Perkins-Kern-Norgren [13], [6]. A constant crack height is assumed in this model. The mathematical description of the dynamic development of the fracture permits its size, the pressure inside the fracture, and stresses at the tips of the fracture to be predicted as a function of time. In particular, the pressure at which breakage begins can be determined from the relationship:

a)
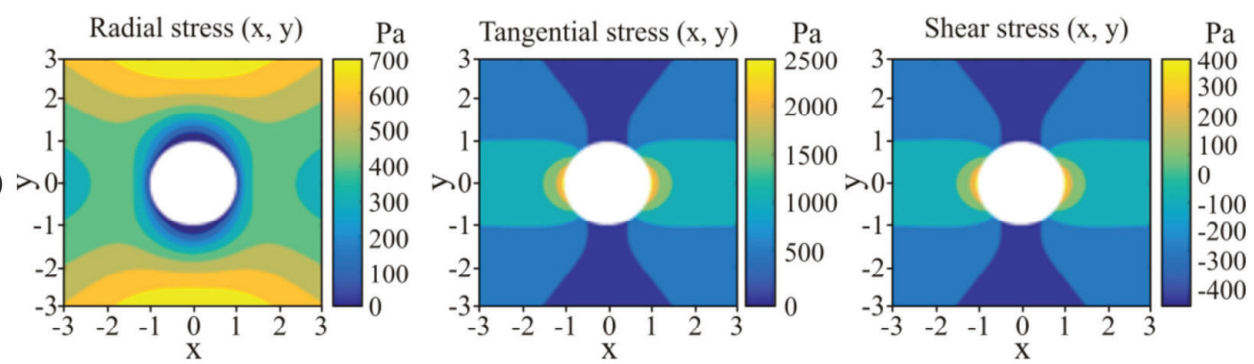

Radial stress $(\mathrm{x}, \mathrm{y})$

Tangential stress $(\mathrm{x}, \mathrm{y})$

+ increse in fluid pressure $\mathrm{Pa}$
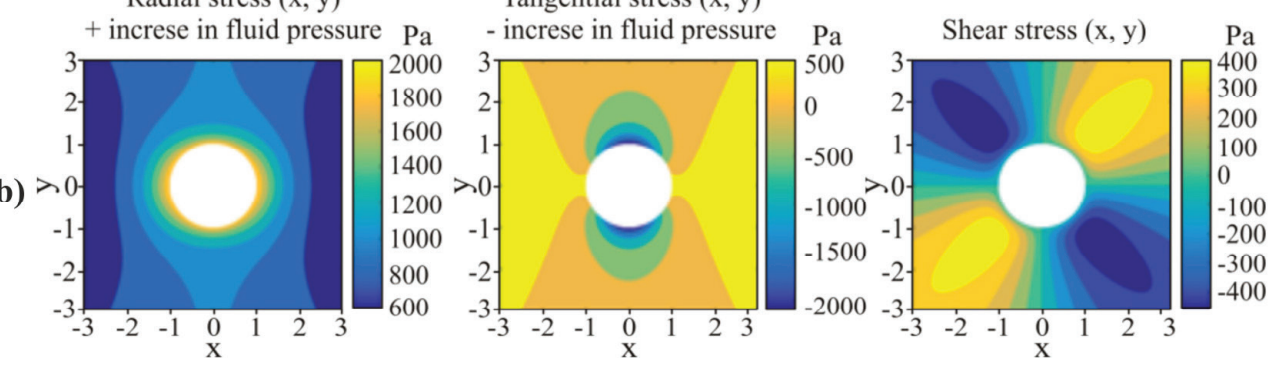

Fig. 3. Stress distribution around the circular opening before and after applying hydraulic pressure a) for a medium without porosity $b$ ) in a porous medium.

a) for a medium without porosity

b) in a porous medium

$$
\mathrm{p}_{b}=3 \sigma_{\min }-\sigma_{\max }+\mathrm{T}-\mathrm{p}_{\mathrm{V}}
$$

$$
\mathrm{p}_{\mathrm{b}}=\frac{3 \sigma_{\text {min }}-\sigma_{\max }+\mathrm{T}-\alpha \frac{1-2 v}{1-v} \mathrm{p}_{v}}{2-\alpha \frac{1-2 v}{1-v}}
$$

$\mathrm{T}-$ tensile strength of the rock, $\sigma_{\min }, \sigma_{\max }-$ minimum and maximum main stress, $\mathrm{p}_{\mathrm{v}}-$ porous pressure, $\alpha$ - Biott coefficient, $v$ - Poisson's ratio. 
Half the length of the crack $\mathrm{L}$ and its width $w$ can be estimated from the formulae:

$$
\mathrm{L}=0.48\left[\frac{8 \mathrm{GQ}^{3}}{(1-\mathrm{v}) \mu}\right]^{1 / 6} \mathrm{t}^{3 / 2} \quad \text { (3) } \quad w=1.32\left[\frac{8(1-\mathrm{v}) \mathrm{Q}^{3} \mu}{\mathrm{G}}\right]^{1 / 6} \mathrm{t}^{1 / 3}
$$

$\mathrm{Q}$ - injection ratio, $\mathrm{G}$ - stiffness coefficient, $\mu$ - hydraulic viscosity, $\mathrm{t}$ - injection time.

This mathematical model, supplemented by the estimation of energy losses resulting from the destruction of the medium around the developing fracture [18], allows one to estimate the fracture growth over time, as is shown in Figure 4 (average crack width is 14 $\mathrm{mm})$.

Now we are coming to the second physically important, geo-mechanical phase of hydrofracturing. Both shear and tensile stresses in the zones surrounding the tips of an advancing fracture [4] can form a cracked zone.The following criterion of micro-crack appearance [15] can be used:

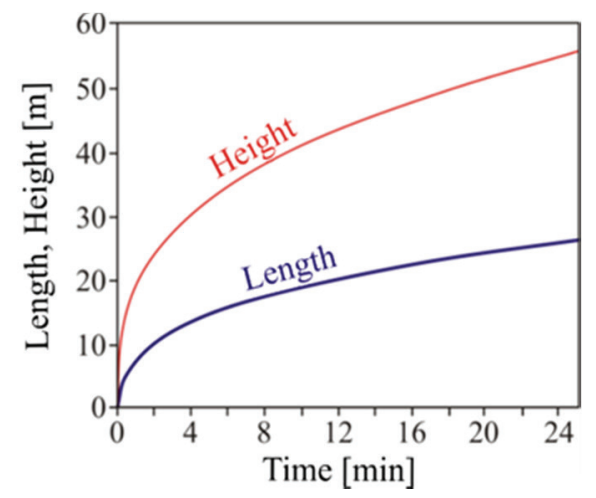

Fig. 4. Height and length of fracture as a function of time during hydro-fracturing [18].

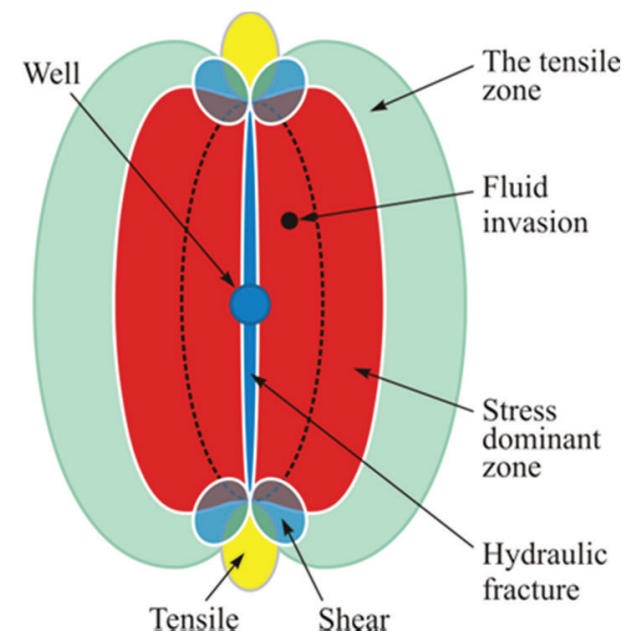

Fig. 5. Development of deformation around a vertical fracture.

$$
\sqrt{\beta^{-1}\left(\sigma_{\mathrm{t}}^{2}+\sigma_{\mathrm{s}}^{2}\right)+\sigma_{\mathrm{n}}^{2}} \geq \mathrm{T}
$$

$\sigma_{\mathrm{s}}, \sigma_{\mathrm{t}}$ and $\sigma_{\mathrm{n}}$ are stresses respectively shear, tangential and normal to the fracture surface, $\mathrm{T}$ is the tensile strength of the rock, $\beta$ is a coefficient depending on the fracture length. 
If a cracked zone is created around the main fracture then a rapid increase in hydraulic permeability can be expected in this zone. With some delay, this zone is saturated with fluid and the saturation changes the stress regime again.

The complicated distribution of stresses, as shown in Fig. 5, alters the physical properties. The differentiation of physical properties has consequences in the differentiation of the electrical resistance and seismic velocity of the rock. These can be recognised by geophysical methods. The distribution of the electromagnetic field was modelled in the surroundings of the hydro-frack and the results are presented in paper [9]. In modelling it was assumed that the porosity $\Phi$ linearly increases with pore pressure p, temperature T and stresses $\sigma$ according to the following formula ( $\mathrm{i}$ is the index of the grid element).

$$
\partial \Phi_{1}=\left(\frac{\alpha_{1}^{2}}{\mathrm{~K}_{1}}+\frac{\alpha_{1}-\Phi_{1}}{\mathrm{~K}_{\mathrm{s}}}\right) \partial \mathrm{p}_{1}+3 \alpha_{\mathrm{T}, 1} \alpha_{1} \partial \mathrm{T}_{\mathrm{i}}+\frac{\alpha_{1}}{\mathrm{~K}_{1}} \partial \sigma_{v}
$$

$\alpha$ is Biota's coefficient, $\alpha_{T}$ is the thermal conductivity coefficient, and $K_{1}$ and $K_{s}$ are the stiffness coefficients of the rock and rock skeleton.

The results of the modelling [9] allow one to assess the development of the electromagnetic field during fracture formation in the hydrofracturing process. If the pores are filled with brine, the results of the electromagnetic measurements are particularly informative.

Numerical modelling of the stresses in a pore-elastic medium has been proposed in [12]. It is based on solving differential equations by approximating them with difference equations and by using the specific capacitive parameters $S_{c}$ and $S_{\varepsilon}$ (formulae 7). The deformation of the rock can be predicted by solution of the parabolic equations under accepted boundary and initial conditions. These deformations can be transformed into changes of physical properties, primarily seismic velocity [3] and electrical resistance.

$$
\mathrm{S}_{\mathrm{c}}=\rho g \frac{\partial \mathrm{p}}{\partial \sigma} \quad \mathrm{S}_{\varepsilon}=\frac{\partial \mathrm{p}}{\partial \varepsilon} \quad \mathrm{S}_{\mathrm{c}}=\rho g \mathrm{~S}_{\varepsilon} \quad \frac{\partial \mathrm{p}}{\partial \mathrm{t}}=\nabla\left[\frac{\phi}{\mathrm{S}_{\mathrm{e}}} \nabla \mathrm{p}\right]+\mathrm{B} \frac{\partial \sigma}{\partial \mathrm{t}} \quad \frac{\partial \mathrm{p}}{\partial \mathrm{t}}=\nabla\left[\frac{\phi}{\mathrm{S}_{\mathrm{e}}} \nabla \mathrm{p}\right]+\frac{\alpha}{\mathrm{S}_{\varepsilon}} \frac{\partial \varepsilon}{\partial \mathrm{t}}(7)
$$

$\Phi$ - permeability, B - Skempton coefficient.

The assumptions under which the models discussed above are constructed make them symmetrical in relation to the main fracture and fully controlled by the volume of injected liquid. To investigate how this model corresponds to reality, seismicity is analysed in the Geysers geothermal field in USA. The Geysers geothermal field is a very extensive deposit located in the subduction zone. A granite intrusion heats up the reservoir water to a temperature of 760 degrees. To stimulate its exploitation, water is injected into 60 boreholes and steam is exploited from 330 wells. A modern seismic network is operating in the area of the deposit, which records about 4,000 shocks per year with a magnitude between 1 and 4.5 .

The results of seismic measurements are processed, mini-earthquake epicentres are located and their seismic moments are estimated. Such data allowed us to examine the distribution of the mini-earthquake epicentres in the area of the Parti 9 injection well. The seismicity recorded between 19 November 2007 and 31 August 2008 (total period) was related to the ratio of water injection. As shown in Figure 6d, from November 2008 to the beginning of April 2008 there was period with a stable injection ratio to the Parti 9 well. After this period, the volume of injected water continuously decreased (decrease period) until the end of the observation period. The relationship between the seismic activity (number of seismic events per unit time) and the volume of injected water ratio was established and can be approximated by the formula shown in Fig. 6c. The seismic activity 
tends to zero when the injection stops (injection is controlling seismicity). The distribution of mini-earthquake epicentres was analysed in two periods, "total" and "decreasing" (Fig. 6a and Fig. 6b).Two conclusions can be drawn from the distributions. The water channels are linear. Decreases in water injection result in a significant decrease in the number of mini-earthquake epicentres in the area, becoming significantly less intensive along the linear channels at larger distances from the well.

a)

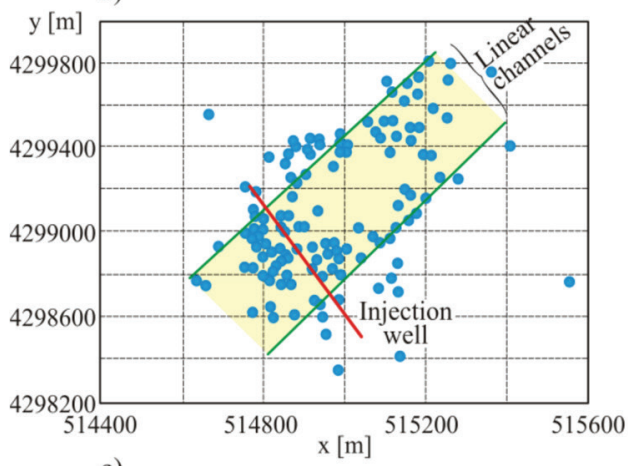

c)

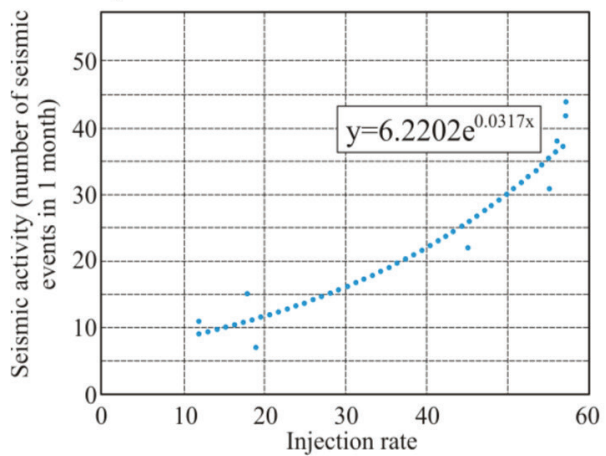

b)

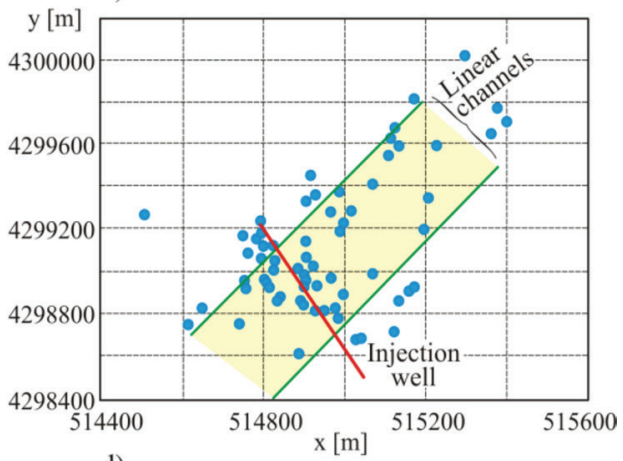

d)

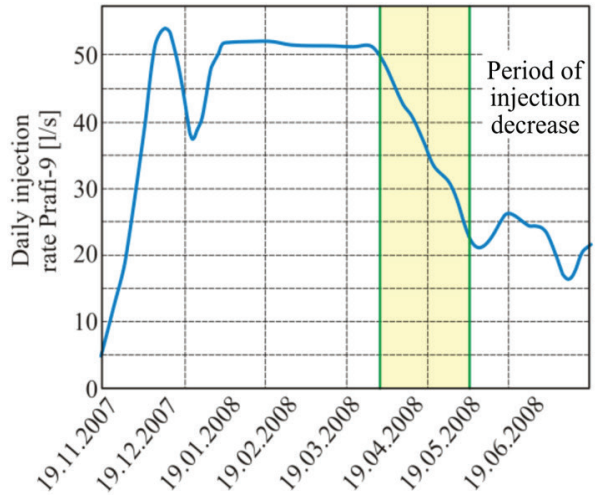

Fig. 6. Location of micro-earthquake epicentres induced by water injection into the Geysers field, USA: a) distribution of micro-earthquake epicentres registered in the period $19^{\text {th }}$ November $2007-31^{\text {st }}$ August 2008, b) distribution of micro-earthquake epicentres registered in a period of decreasing volume of water injection (marked in figure d), c) relationship between seismic activity and the volume of water injection ratio, d) change of water injection ratio with time.

The liquid flow along linear elements is different from that in volume. Hydraulic resistance to linear flow and its development over time can be described by the following formula from Bear et al. [2]:

$$
\phi_{\mathrm{i}}=\frac{1}{12} \frac{\rho \mathrm{gL}}{\mu} \int_{0}^{\infty} \mathrm{b}^{3} \mathrm{f}(\mathrm{b}) \mathrm{db} \quad-\frac{\partial}{\partial \mathrm{x}_{\mathrm{i}}}\left(\phi_{\mathrm{i}} \frac{\partial \varphi}{\partial \mathrm{x}_{\mathrm{i}}}\right)=\mathrm{S}_{\mathrm{i}} \frac{\partial \varphi}{\partial \mathrm{t}} \quad \varphi=\mathrm{z}+\frac{\mathrm{p}}{\mathrm{g} \rho}
$$

$\mathrm{L}$ - length of the linear element, $\mathrm{b}$ - width of the linear element described by probability distribution $\mathrm{f}$ (b), $\mu$ - viscosity of the fluid, $\mathrm{Si}$ - hydraulic capacity of the medium, $\mathrm{z}$ - depth, $\mathrm{g}$ - gravity constant, $\rho$ - density, $p$ - hydraulic pressure.

These equations indicate that, at a small hydraulic capacity of the geological medium, the hydraulic pressure at the ends of the linear elements, even at long distances, increases significantly over time, especially when the channels are widened by non-elastic 
deformations caused by hydraulic pressure.

It can be concluded that one needs more information to model stresses, deformations and fracturing as a result of hydro-fracturing in an anisotropic medium with linear hydraulic channels and so therefore it is practically impossible. Geophysical observations using seismology and seismic interferometery methods can be helpful in assessing the risk of the dangerous hydrofracturing developments.

Griffith's generalised theory may be helpful in interpreting the seismological observations. The total energy $\Delta$ Freleased by the formation of $\mathrm{N}$ cracking can be estimated as [18]:

$$
\Delta \mathrm{F}=\mathrm{Nw}\left(-\mathrm{B}^{2} \sigma^{2}\left\langle\mathrm{c}^{2}\right\rangle+4 \gamma\langle\mathrm{c}\rangle\right)
$$

$\langle c\rangle$ is the expected value of a half-length of the crack, $\sigma$ - the tensile stress in the element of medium B is constant depending on the elastic properties of the medium, $w$ is the width of the block in which the cracks are formed and $2 \gamma$ is the energy needed to separate the cracked surface.

A coefficient $G=\frac{\partial \Delta F}{\partial A}$ of energy relaxation per unit increment of free fracture surface can be introduced, where $\mathrm{A}=4 \mathrm{cw}$. This parameter determines the rate of growth of the cracked area [10] and if it is near the critical value then its increase may be noticeable in the measurement data, both seismological and seismic [5]. Ouchi in [11], shows that an increase in seismic events $d n(t)$ depends on the size of the population $n(t)$ and the coefficients of growth and also depends on a h(t) representing local conditions:

$$
\begin{aligned}
& \operatorname{dn}(\mathrm{t}) / \mathrm{dt}=\mathrm{n}(\mathrm{t})\left[\alpha-\beta \mathrm{n}(\mathrm{t})-\int_{-\infty}^{\mathrm{t}} \mathrm{n}(\mathrm{s}) \mathrm{h}(\mathrm{t}-\mathrm{s}) \mathrm{ds}\right] \\
& R(t)=\frac{\int_{t_{i}-t_{s}}^{t_{i}+t_{s}} f_{\text {obs }}\left(t^{\prime}+t_{i}\right) f_{\text {ref }}\left(t^{\prime}\right) d t^{\prime}}{\int_{t_{i}-t_{s}+t_{s}}^{t_{s}} f^{2}{ }_{\text {obs }}\left(t^{\prime}\right) d t^{\prime} \int_{t_{i}-t_{s}}^{t_{i}+t_{s}} f_{\text {ref }}{ }^{2} \text { ref }\left(t^{\prime}\right) d t^{\prime}}
\end{aligned}
$$

Approaching a critical situation means an increase in the growth coefficient so that, despite the reduction of the main cause of the seismicity, the volume of injection fluid, seismic activity does not decrease or even increase. On the other hand, with the seismic interferometry method, the seismic velocity distribution in the geological medium can be recognised from the seismic noise recorded.

If the medium is under significant non-elastic deformation over time, then changes in the seismic velocity distribution are directly related to the deformations. The stretching coefficient (11), [7] the maximum of the correlation function calculated from the seismic noise recorded in different time windows, can control the deformation caused by hydrofracturing.

\section{Summary}

The deformation process in a geological medium due to hydrofracturing is complicated. However some of its phases can be modelled on the basis of the algorithms presented in the article. Such modelling can be useful in planning and arranging the geophysical measurements, such as those obtained by seismological and seismic interferometry 
methods. The results of such measurements, together with data from other methods, can help in controlling the risk of dangerous rock deformations due to hydrofracturing.

This work was carried out in the framework of the Shale Gas Exploration and Exploitation Induced Risks (SHEER) project funded by the European Union's Horizon 2020 Research and Innovation Programme under grant agreement no. 640896.

\section{References}

1. BCOGC, Investigation of induced seismicity in the Horn River Basin (British Columbia Oil and Gas Commission open report, 2012)

2. J. Bear, C.F. Tsang, G. De Marsily, Flow and contaminat transport in fractured rock (Academic Press, 1993)

3. C. Brisco, M. van der Baan. A review of seismic velocity response to variatiation in pore pressure pore-saturating fluid and confirming stress (GeoConvection Calgary, Canada, 2016)

4. C. Cipolla, S. Maxwell, M. Mack, R. Downie, A practical guide to interpreting microseismic measurements (SPE North American Unconventional Gas Conference and Exhibition, 2011)

5. R. Czarny, H. Marcak, N. Nakata, Z Pilecki Z. Isakow, Pure. Appl. Geophys. 173, 6, 1907-1916 (2016) doi 10.1007/s00024-015-1234-3

6. J. Geertsma, J.F. de-Klerk, J. Petrol. Technol. 21, 12, 1571-1581 (1969) doi 10.2118/2458-PA

7. C. Hadzioannou, E. Larose O. Coutant P. Roux, J. Acoust. Soc. Am. 125, 6, 36883695 (2009) doi: 10.1121/1.3125345

8. G. Hillers, S. Husen, A. Obermann, T. Planes, E. Laroce, M Campillo, Noise-based monitoring and imaging of a seismic transient deformation induced by the 2006 Basel reservoir stimulation, Geophysics 80, 4, 51-68 (2015)

9. J. Kim, E. Schankee Um, J.G. Moridis, Fracture propagation, fluid flow, and geomechanics of water-based hydraulic fracturing in shale gas systems and electromagnetic geophysical monitoring of fluid migration (SPE 168578, Hydraulic Fracturing Technology Conference, Texas/USA, 2014)

10. I. G. Main, A modified Griffith criterion for the evolution of damage with a fractal distribution of crack lengths: application to seismic event rates and b-values, Geophys. J. Int. 107, 353-362 (1991)

11. T. Ouchi, Pure. Appl. Geophys. 140, 1, 15-28 (1993) doi 10.1007/BF00876868

12. V. Palciauskas, P.A. Domenico, Water. Resour. Res. 25, 2, 203-213 (1989) doi 10.1029/WR025i002p00203

13. T.K. Perkins, L.R. Kern, J. Petrol. Technol. 13, 9, 937-949 (1961) doi 10.2118/89-PA

14. J. Perrin, T. Cook, Hydraulically fractured wells provide two-third of US national gas production US Energy (Independent Statistics and Analysis U.S. Energy Information Administration, 2016)

15. G. Ruiz, M. Ortiz, A. Pandolfi, Three-dimensional finite-element simulation of the dynamic Brazilian tests on concrete cylinders, Int. J. Numer. Meth. Eng. 48, 963-994 (2000)

16. D.R. Schmitt, M.D. Zoback, Int. J. Rock. Mech. Min. 26, 6, 499-506 (1989) doi 10.1016/0148-9062(89)91427-7 
17. C.H. Scholz, The mechanics of earthquakes and faulting (Cambridge University Press, 1990)

18. P. Valko, M.J. Economides, Propagation of hydraulically induced fractures - a continuum damage mechanic approach, Int. J. Rock. Mech. Min. 31, 3, 221-229 (1994) 